\title{
Still's Disease Mimicking Sepsis in a Young Woman: A Case Report and Literature Review
}

\author{
Maneesh Gaddam ${ }^{1 *}$, MD, Olga Alexandra Reyes ${ }^{2}$, Cosmina Zeana ${ }^{3}$, Gilda Diaz-Fuentes ${ }^{4}$, \\ 1Pulmonary and Critical Care Medicine BronxCare Health System 1650 Grand Concourse, Bronx, NY 10457 Affiliated with Icahn School of \\ Medicine at Mount Sinai, \\ ${ }^{2}$ Internal Medicine resident Department of Internal Medicine BronxCare Health System 1650 Grand Concourse, Bronx, NY 10457 Affiliated with \\ Icahn School of Medicine at Mount Sinai, \\ ${ }^{3}$ Assistant Professor of Clinical Medicine Division of Infectious Diseases BronxCare Health System1650 Grand Concourse, Bronx, NY 10457 \\ Affiliated with Icahn School of Medicine at Mount Sinai, \\ ${ }^{4}$ Associate Professor of Clinical Medicine Division of Pulmonary and Critical Care Medicine BronxCare Health System 1650 Grand Concourse, \\ Bronx, NY 10457 Affiliated with Icahn School of Medicine at Mount Sinai,
}

Corresponding Author: Maneesh Gaddam, Pulmonary Fellow Division of Pulmonary and Critical Care Medicine BronxCare Health System 1650 Grand Concourse, Bronx, NY 10457 Affiliated with Icahn School of Medicine at Mount Sinai,

Received Date: October 11, 2020; Accepted Date: November 02, 2020; Published Date: November 09, 2020. Citation: Maneesh Gaddam., Olga A. Reyes., Cosmina Zeana., Gilda D. Fuentes, (2020) Still's disease mimicking sepsis in a young woman. A case report and literature review. J Clinical Case Reports and Studies, 1(6): Doi:10.31579/2690-8808/022

Copyright: (C) 2020 Maneesh Gaddam, This is an open-access article distributed under the terms of the Creative Commons Attribution License, which permits unrestricted use, distribution, and reproduction in any medium, provided the original author and source are credited.

\section{Abstract \\ Background}

Adult onset Still's disease (ASD) is a rare systemic auto-inflammatory condition that often poses a diagnostic dilemma due to nonspecific clinical signs and symptoms. We report a case of ASD that dramatically improved after a short course of steroids.

Case presentation A 23-year-old women from Ghana presented with a two-week history of fever, sore throat, right-sided neck swelling and an evanescent, pruritic rash on her extremities. On exam she was febrile to $103 \mathrm{~F}$, tachycardic, tachypneic, had tender, right-sided cervical lymphadenopathy, and diffuse joint tenderness with decreased range of motion. She was admitted to the ICU for possible sepsis and started on broad-spectrum antibiotics. Extensive work-up for possible infectious and auto-immune etiologies, including a bone marrow biopsy was negative. The diagnosis of Still's disease was made based on the Yamaguchi criteria. The patient was started on methyl-prednisolone and significantly improved within 24 hours.

Conclusion

Patients presenting with ASD often represent a diagnostic challenge. Clinicians should be aware of this rare disorder, exclude other more frequent diseases and start early anti-inflammatory therapy to avoid poor outcomes.

Keywords: adult onset Still's disease; polyarteritis nodosa; macrophage activation syndrome

\section{Introduction:}

Adult Still's disease (ASD) is a rare systemic auto inflammatory condition first described in children by Dr. George Still in 1896 as an entity similar to adult rheumatoid arthritis with associated splenomegaly and lymphadenopathy.[1] A similar condition with an evanescent maculopapular rash associated with fever usually in the evening, arthritis mainly affecting the knees, fingers, and wrists was later described by Bywaters. Associated features included splenomegaly, pleural effusions, and pericarditis.[2]

The exact incidence and prevalence of adult onset Still's disease is unclear. Multiple studies suggest a higher incidence in women usually older than 35 years/age. A retrospective study of 62 cases in West France from 19821991, reported a disease incidence calculated over five years of $0.16 / 100,000$. The mean age was 36 years, with two peaks of distribution at 15-25 and 36-45 years.[3] Other studies reported an estimated annual incidence of $0.4-0.62 / 100,000$ adults and a prevalence of 3.46.77/100,000.[4,5] We present a young patient with features of sepsis which was subsequently diagnosed with ASD.

\section{Case Presentation}

A 23-year-old female from Ghana with no past medical history or toxic habits was admitted to the intensive care unit with fever, tachycardia, and leukocytosis. The initial diagnosis was sepsis and she was started on broad-spectrum antibiotics. The patient has been living in the United States for the past 3 years after moving from Ghana, but she traveled to Kansas about 1 month prior to the presentation where she had unprotected sexual encounter with one partner.

Patient reported painful right sided neck swelling of two weeks duration. 
Soft tissue ultrasound of the neck revealed multiple right-sided lymph nodes, the largest measuring $1.2 \times 0.8 \times 1.0 \mathrm{~cm}$ in size. Initially, she was given non-steroidal anti-inflammatory (NSAIDS) medications and sent home. She returned to the hospital within 24 hours with pruritic erythematous rashes of upper and lower extremities, severe persistent neck swelling and pain associated with high fever and sore throat which prompted intensive care admission for possible sepsis.

On exam, she was tachycardic with a heart rate of 140/min, febrile with a temperature of $102{ }^{\circ} \mathrm{F}$, tachypneic with a respiratory rate of $25 / \mathrm{min}$ and normotensive. There were tender right neck lymphadenopathy and bilateral tonsillar erythema, no stridor. Lungs were clear, no heart murmur, and no visceromegaly in the abdominal exam. Diffuse joint tenderness with a range of motion but no swelling or fluid was seen. Neurological and skin exam was normal. Computed tomogram of the neck only revealed small

\begin{tabular}{|c|c|c|c|c|}
\hline & $\begin{array}{c}\text { On admission } \\
\text { (Day 1) }\end{array}$ & $\begin{array}{r}\text { Prior to } \\
\text { steroids } \\
\text { (Day 7) } \\
\end{array}$ & $\begin{array}{l}24 \text { Hours after } \\
\text { steroids }\end{array}$ & $\begin{array}{l}\text { Follow up } \\
\text { (Day 36) }\end{array}$ \\
\hline $\begin{array}{l}\text { Complete Blood Count: } \\
\text { Hemoglobin: }(\mathrm{g} / \mathrm{dl}) \\
\text { White call count: }(\mathrm{k} / \mathrm{ul}) \\
\text { Neutrophils: }(\%) \\
\text { Platelets: }(\mathrm{k} / \mathrm{ul})\end{array}$ & $\begin{array}{l}11.8 \\
12.8 \\
86.2 \\
242\end{array}$ & $\begin{array}{c}9.8 \\
22.5 \\
92.1 \\
289\end{array}$ & $\begin{array}{c}9.1 \\
22.8 \\
19 \\
492\end{array}$ & $\begin{array}{c}8.8 \\
8.4 \\
73.3 \\
450\end{array}$ \\
\hline $\begin{array}{l}\text { Basic Metabolic Panel: } \\
\text { Sodium: }(\mathrm{mEq} / \mathrm{L}) \\
\text { Potassium: }(\mathrm{mEq} / \mathrm{L}) \\
\text { BUN: }(\mathrm{mg} / \mathrm{dl}) \\
\text { Creatinine: }(\mathrm{mg} / \mathrm{dl})\end{array}$ & $\begin{array}{c}140 \\
4.2 \\
6 \\
0.5\end{array}$ & $\begin{array}{c}135 \\
4.5 \\
8 \\
0.6\end{array}$ & $\begin{array}{c}135 \\
4.2 \\
7 \\
0.4\end{array}$ & $\begin{array}{c}141 \\
4.4 \\
4 \\
0.5\end{array}$ \\
\hline $\begin{array}{l}\text { Liver function tests: } \\
\text { Alanine } \\
\text { aminotransferase: } \\
\text { (units/L) } \\
\text { Aspartate } \\
\text { aminotransferase: } \\
\text { (units/L) } \\
\text { Alkaline phosphatase: } \\
\text { (units/L) } \\
\text { Total Bilirubin: (mg/dl) }\end{array}$ & $\begin{array}{l}19 \\
29 \\
48 \\
0.2\end{array}$ & $\begin{array}{l}50 \\
63 \\
97 \\
0.4\end{array}$ & $\begin{array}{c}66 \\
47 \\
103 \\
0.2\end{array}$ & $\begin{array}{r}38 \\
56 \\
73 \\
0.2\end{array}$ \\
\hline $\begin{array}{l}\text { Other labs: } \\
\text { Erythrocyte } \\
\text { Sedimentation Rate: } \\
\text { (mm/hr) } \\
\text { C-Reactive Protein: } \\
\text { (mg/L) } \\
\text { Ferritin: (ng/ml) }\end{array}$ & $\begin{array}{c}22 \\
67.74 \\
>5000\end{array}$ & $\begin{array}{c}64 \\
273.89 \\
>5000\end{array}$ & $\begin{array}{c}58 \\
99.35\end{array}$ & $\begin{array}{c}73 \\
16.80 \\
4483\end{array}$ \\
\hline $\begin{array}{l}\text { Vital signs: } \\
\text { Heart Rate: }(\mathrm{bpm}) \\
\text { Temperature: }\left({ }^{\circ} \mathrm{F}\right)\end{array}$ & $\begin{array}{c}140 \\
101.8\end{array}$ & $\begin{array}{l}138 \\
103\end{array}$ & $\begin{array}{c}92 \\
97.5\end{array}$ & $\begin{array}{r}105 \\
98.7\end{array}$ \\
\hline
\end{tabular}

Table 1: Pertinent Laboratory: 1 .

right-sided lymphadenopathy. Chest-X-ray, electrocardiogram, echocardiogram, abdominal ultrasound, urine toxicology, HIV test, blood and urine cultures, chlamydia, gonorrhea, and syphilis testing, as well as hepatitis $\mathrm{A}, \mathrm{B}$ and $\mathrm{C}$ were all negative. Rheumatoid factor was slightly positive $(16.4 \mathrm{IU} / \mathrm{mL})$, aldolase $(28.9 \mathrm{U} / \mathrm{L})$, ferritin $(>5,000 \mathrm{ng} / \mathrm{ml})$ and serum lactic dehydrogenase (658 unit/L) were elevated. Malaria smear in three consecutive days was negative. Autoimmune workup revealed negative anti-SCL 70, antinuclear, anti-CCP and anti-centromere antibodies. Serology revealed history of exposure to Epstein Barr and Cytomegaloviruses, negative Parvovirus B19 IgM and IgG antibodies. Serology for Dengue, Chikungunya, Zika, Leptospira and Lyme's disease was negative. Pertinent laboratory during admission can be seen in Table

Patient's condition continued to deteriorate despite fluids and broadspectrum antibiotics including Piperacillin-Tazobactam, Vancomycin, Doxycycline. She was weak, febrile, tachycardic, and unable to move due to arthralgias and myalgias. Other differentials considered were adult Still's disease, leukemia and lymphoma. Bone marrow biopsy was performed on day 6 of admission and patient was started on methylprednisolone; within 24 hours she became afebrile with resolution of tachycardia and tachypnea. She had a steady clinical recovery and within 3-4 days she was close to normal. Bone marrow biopsy reported a normocellular marrow with $70 \%$ cellularity which ruled out hemophagocytic syndrome, leukemia or lymphoma, leaving Still's disease as the most possible diagnosis.

\section{Discussion}

The clinical presentation of ASD is non-specific; the triad of fever, evanescent rash and arthralgia/arthritis is the commonest presentation. Fever is seen in nearly $100 \%$ of patients, with the temperature being greater than $39^{\circ} \mathrm{C}$. One study of 45 patients with ASD showed that only $43 \%$ of patients presented with the typical triad; fever was present in all patients, arthralgias in $98 \%$ and rash in $82 \%$ of the patients.[6] Physical exam is equally nonspecific. Another study with 62 patients, reported higher frequencies of sore throat $(92 \%)$, weight loss $(76 \%)$, lymphadenopathy $(74 \%)$, pleuritis $(53 \%)$, pneumonitis $(27 \%)$, abdominal pain (48\%), hepatomegaly (44\%) or abnormal liver function tests (76\%)". Liver failure occurred in conjunction with the use of aspirin and NSAID use.[7] An elevated erythrocyte sedimentation rate (ESR) (100\%) and leukocytosis (93.8\%) were findings identified in a Thai study of ASD.[08]

In 1992, the Yamaguchi criterion was structured to improved identification 
and diagnosis of ASD. This criterion carries a sensitivity of 93.5\%.[09] There are four major criteria which include high fever $\left(39^{\circ} \mathrm{C}\right)$, rash (evanescent salmon-pink colored, which generally appear in association with fever), arthralgia/arthritis and leukocytosis. Minor criteria are sore throat, lymphadenopathy, hepatomegaly and/or splenomegaly, elevated liver function enzymes and negative rheumatoid factor and anti-nuclear antibody.[10] The patient should meet at least 5 of the above-mentioned criteria, out of which 2 must be major for the diagnosis of ASD to be considered.

There is no specific test to confirm the diagnosis of ASD, which represent a challenge to the clinicians as many features of ASD can be seen in other more common conditions like sepsis, systemic inflammatory response syndrome, autoimmune disorders, hemophagocytic lymphohistiocytosis (HLH) and malignancy. Acute phase reactants such as ferritin, ESR, Creactive protein (CRP) will be elevated; a ferritin levels greater than 3,000 $\mathrm{ng} / \mathrm{mL}$ can be observed and if levels are greater than $10,000 \mathrm{ng} / \mathrm{mL}$, presence of co-existing HLH should be evaluated.[11] In cases where bone marrow biopsy is available, presence of peripheral neutrophilia, granulocyte hyperplasia and hypercellularity can be seen which helps to differentiate from the pancytopenia characteristic of hemophagocytic syndrome.

Infectious etiologies to be consider includes viral hepatitis, acute HIV infection, parvovirus B19, mosquito-borne viral infections (such as Zika and Chikungunya viruses), Epstein-Barr, Cytomegalovirus, coxsackie virus, mumps, rubella, Lyme's disease. Serology is helpful to exclude some of these diagnoses.

Rheumatologic conditions such as rheumatoid arthritis, systemic lupus erythematosus, and reactive arthritis all have similar presentation; serology will help to narrow down diagnosis as the ANA, RF, anti-CCP negative and anti-dsDNA will be negative in ASD. Among the vasculitis disorders, polyarteritis nodosa (PAN) has the most similarities; however, the rash seen in PAN is purpuric with ulcerative lesions. Autoimmune serology, biopsy of an affected organ and vascular imaging studies can be useful for diagnosis. In cases where lymphomas and leukemias are been considered, peripheral smear and bone marrow biopsy will be helpful.

An important hematologic entity to be considered is hemophagocytic lymphohistiocytosis (HLH); also known as macrophage activation syndrome (MAS) when associated with ASD and other rheumatic disorders. Typically, it will present as high-grade fever, new hepatosplenomegaly, lymphadenopathy, pancytopenia or bicytopenia (including low platelet counts) and abnormal liver enzymes; some patients can develop neurologic disturbances and bleeding disorder. Similar to ASD, markedly elevated ferritin level is also present. [12] Diagnosis should be suspected with changes in the clinical course of a patient with active rheumatic disease. Bone marrow biopsy will not always show the presence of hemophagocytes, especially if obtained early in the disease presentation.

Imaging in early phases of ASD is generally non-contributive in establishing a diagnosis. Radionuclide bone scan and gadolinium-enhanced magnetic resonance imaging might be more sensitive imaging for early diagnosis and successful treatment in follow up. A study comparing the imaging of patients with ASD, juvenile arthritis and adult-onset rheumatoid arthritis revealed pericapitate articular alterations without radiocarpal involvement to be frequent in the setting of ASD.[13] An unusual complication of ASD is the rapid destruction of the hip and, less commonly, the knee, which can require total joint arthroplasty. [14]

Our patient met all four major and four minor criteria; she had persistent high fever, severe sore throat, lymphadenopathy, and leukocytosis with elevated ESR. She had an evanescent rash and transaminitis at some point during the clinical course. Laboratory was not suggestive of HLH and Auctores Publishing - Volume 2(1)-24 www.auctoresonline.org ISSN: $2690-880$ serology, septic work up and bone marrow biopsy excluded other diagnoses.

The mainstay management of ASD is supportive, with fluids and antiinflammatory medications. In general, NSAIDs are to be tried first, but studies show that it provides remission and disease control in only $21 \%$ of patients. [15] Multiple studies reveal that corticosteroids are effective in controlling the disease by up to $60 \%$. [6,7,15,16]. Addition of steroidsparing agents early in the course of the disease, in a patient that has not completely responded to steroids can decrease side effects and the dependence of steroids. Multiple disease modifying anti-rheumatic drugs have been studied in the management of ASD; methotrexate is the first line steroid-sparing treatment that can potentially prevent worsening of arthritis. During treatment with steroids, ESR, CRP and glycosylated ferritin levels should be checked to monitor response; some patients who initially shows a response can develop splenomegaly, persistently elevated ESR and low glycosylated ferritin which suggest consideration to use steroids sparing agents. [17]

In a French study of 20 patients with ADS requiring ICU care, corticosteroids were given as the first line, with an efficacy of $50 \%$. Anakinra, a biologic drug that helps to decrease inflammation in rheumatoid arthritis and other inflammatory diseases proved to be the most successful by being effective in $80 \%$ of the patients with ADS. [18] Immunosuppressants and biologic agents have been studied and proven effective in the maintenance phase of the disease.

Patients with ASD can have elevated levels of cytokines such as interleukin (IL)-1, IL-6, interferon (IFN)-gamma, and tumor necrosis factor-alpha which are thought to be involved in the pathogenesis of the disease. Azathioprine, cyclosporine A, cyclophosphamide and leflunomide have proven efficacious as corticosteroid-sparing drugs in patients with multiple joint affected course of the disease.[19] Interleukin 1 (IL-1) antagonists have been used as third-line treatment (after corticosteroids and immunosuppressants have failed or had severe side effects) with promising results. Anakinra has been the main drug studied and proved to be not only effective in about $80 \%$ of the cases, but also has provided remission of disease after 3 months of use.[20] In the setting of IL-6 antagonists, Tocilizumab has been studied as a single agent in corticosteroid and methotrexate refractory ASD. Treatment duration was for 12 months and patients were observed for 18 months; resolution of fever and joint manifestations as well as decrease in ESR, CRP and ferritin levels, was observed with continued use of medication, making it a promising alternative to refractory disease.[21]

Outcomes of patients with ASD falls in three possible courses of the disease: self-limited, intermittent flares and chronic disease, with about one-third falling in each category. Most of the patients with self-limited disease achieve remission in about 9 months. Patients with the intermittent disease develop recurrent episodes of flares with complete remission of symptoms between flares. Those with the chronic disease develop progressively worsening joint destruction.[22]

In conclusion, patients presenting with adult onset of Still's disease represent a challenge to the clinicians. A wide range of clinical presentation and severity of illness can be seen across all medical services, ranging from ambulatory setting to critical care. It is of the utmost importance to keep in mind the existence of this rare disorder, exclude other common and deadly conditions rapidly and ideally start early antiinflammatory treatment to avoid poor outcomes.

\section{Conflict of Interest:}

The authors have no conflict of interest to declare

Abbreviations:

ASD: Adult Still's disease 
CRP: C-reactive protein

ESR: Erythrocyte sedimentation rate

HLH: Hemophagocytic lymphohistiocytosis

MAS: Macrophage activation syndrome

NSAIDS: Nonsteroidal anti-inflammatory drugs.

PAN: Polyarteritis nodosa

\section{References.}

1. Still GF. On a Form of Chronic Joint Disease in Children. Medico-chirurgical transactions. 1897;80:47-60.49.

2. Bywaters EG. Still's disease in the adult. Annals of the rheumatic diseases. 1971;30(2):121-133.

3. Magadur-Joly G, Billaud E, Barrier JH, et al. Epidemiology of adult Still's disease: estimate of the incidence by a retrospective study in west France. Annals of the rheumatic diseases. 1995;54(7):587-590.

4. Evensen KJ, Nossent HC. Epidemiology and outcome of adult-onset Still's disease in Northern Norway. Scandinavian journal of rheumatology. 2006;35(1):48-51.

5. Balci MA, Pamuk ON, Pamuk GE, Uzundere FK, Donmez S. Epidemiology and outcome of adult-onset Still's disease in Northwestern Thrace region in Turkey. Clinical and experimental rheumatology. 2015;33(6):818-823.

6. Wouters JM, van de Putte LB. Adult-onset Still's disease; clinical and laboratory features, treatment and progress of 45 cases. The Quarterly journal of medicine. 1986;61(235):1055-1065

7. Pouchot J, Sampalis JS, Beaudet F, et al. Adult Still's disease: manifestations, disease course, and outcome in 62 patients. Medicine. 1991;70(2):118-136.

8. Louthrenoo W, Aramsareewong T, Sukitawut W. Adult onset Still's disease: clinical features and outcome in 16 Thai patients. Journal of clinical rheumatology : practical reports on rheumatic \& musculoskeletal diseases. 2001;7(5):301-307.

9. Masson C, Le Loet X, Liote F, et al. Comparative study of 6 types of criteria in adult Still's disease. The Journal of rheumatology. 1996;23(3):495-497.

10. Yamaguchi M, Ohta A, Tsunematsu T, et al. Preliminary criteria for classification of adult Still's disease. The Journal of rheumatology. 1992;19(3):424-430.

11. Coffernils M, Soupart A, Pradier O, Feremans W, Neve P, Decaux G. Hyperferritinemia in adult onset Still's disease and the hemophagocytic syndrome. The Journal of rheumatology. 1992;19(9):1425-1427.

12. Lerkvaleekul B, Vilaiyuk S. Macrophage activation syndrome: early diagnosis is key. Open access rheumatology : research and reviews. 2018;10:117-128.

13. Bjorkengren AG, Pathria MN, Sartoris DJ, et al. Carpal alterations in adult-onset Still disease, juvenile chronic arthritis, and adult-onset rheumatoid arthritis: comparative study. Radiology. 1987;165(2):545-548.

14. Cabane J, Michon A, Ziza JM, et al. Comparison of long term evolution of adult onset and juvenile onset Still's disease, both followed up for more than 10 years. Annals of the rheumatic diseases. 1990;49(5):283-285

15. Gerfaud-Valentin M, Maucort-Boulch D, Hot A, et al. Adult-onset still disease: manifestations, treatment, outcome, and prognostic factors in 57 patients. Medicine. 2014;93(2):91-99.

16. Gopalarathinam R, Orlowsky E, Kesavalu R, Yelaminchili S. Adult Onset Still's Disease: A Review on Diagnostic Workup and Treatment Options. Case Rep Rheumatol. 2016;2016:6502373.

17. Jamilloux $\mathrm{Y}$, Gerfaud-Valentin M, Henry T, Seve P. Treatment of adult-onset Still's disease: a review. Ther Clin Risk Manag. 2015;11:33-43.

18. Neel A, Wahbi A, Tessoulin B, et al. Diagnostic and management of life-threatening Adult-Onset Still Disease: a French nationwide multicenter study and systematic literature review. Critical care (London, England). 2018;22(1):88

19. Efthimiou P, Georgy S. Pathogenesis and management of adult-onset Still's disease. Seminars in arthritis and rheumatism. 2006;36(3):144-152.

20. Moulis G, Sailler L, Astudillo L, Pugnet G, Arlet P. May anakinra be used earlier in adult onset Still disease? Clinical rheumatology. 2010;29(10):1199-1200.

21. Ortiz-Sanjuan F, Blanco R, Calvo-Rio V, et al. Efficacy of tocilizumab in conventional treatment-refractory adult-onset Still's disease: multicenter retrospective open-label study of thirty-four patients. Arthritis \& rheumatology (Hoboken, N.J.). 2014;66(6):1659-1665.

22. Efthimiou P, Paik PK, Bielory L. Diagnosis and management of adult onset Still's disease. Annals of the rheumatic diseases. 2006;65(5):564-572. 